\title{
The CBP/p300 TAZ1 domain in its native state is not a binding partner of MDM2
}

\author{
Theresia MATT, Maria A. MARTINEZ-YAMOUT, H. Jane DYSON and Peter E. WRIGHT ${ }^{1}$ \\ Department of Molecular Biology and the Skaggs Institute for Chemical Biology, The Scripps Research Institute, 10550 North Torrey Pines Road, La Jolla, CA 92037, U.S.A.
}

\begin{abstract}
The transcriptional co-activator CBP [CREB (cAMP-responseelement-binding protein)-binding protein] and its paralogue $\mathrm{p} 300$ play a key role in the regulation of both activity and stability of the tumour suppressor p53. Degradation of p53 is mediated by the ubiquitin ligase MDM2 (mouse double minute protein) and is also reported to be regulated by $\mathrm{CBP} / \mathrm{p} 300$. Direct protein-protein interaction between a central domain of MDM2 and the TAZ1 (transcriptional adaptor zinc-binding domain) [C/H1 (cysteine/ histidine-rich region 1)] domain of p300 and subsequent formation of a ternary complex including p53 have been reported previously. We expressed and purified the proposed binding domains of HDM2 (human homologue of MDM2) and CBP, and examined their interactions using $C D$ spectroscopy. The binding studies were extended by using natively purified GST (glutathione S-transferase)-p300 TAZ1 and GST-p53 fusion proteins, together with in vitro translated HDM2 fragments, under similar solution
\end{abstract}

conditions to those in previous studies, but omitting added EDTA, which causes unfolding and aggregation of the zinc-binding TAZ1 domain. Comparing the binding properties of the known TAZ1 interaction partners HIF-1 $\alpha$ (hypoxia-inducible factor 1), CITED2 $(\mathrm{CBP} / \mathrm{p} 300$-interacting transactivator with glutamic- and asparticrich tail) and STAT2 (signal transducer and activator of transcription 2) with HDM2, our data suggest that TAZ1 in its native state does not serve as a specific recognition domain of HDM2. Rather, unfolded TAZ1 and HDM2 proteins have a high tendency to aggregate, and non-specific protein complexes are formed under certain conditions.

Key words: cAMP-response-element-binding-protein-binding protein (CBP), glutathione $S$-transferase pull down, oncoprotein, mouse double minute protein (MDM2), protein secondary structure, zinc finger.

\section{INTRODUCTION}

CBP [CREB (cAMP-response-element-binding protein)-binding protein $] / \mathrm{p} 300$ are members of a family of transcriptional coactivators which are involved in cell growth, development, and differentiation [1-4]. Most of their known functions are mediated by protein-protein interactions through highly conserved domains; the number of described $\mathrm{CBP} / \mathrm{p} 300$ interaction partners is still growing, and many different ligands have been found to interact with a given co-activator domain. It is assumed that binding proteins compete for limited amounts of $\mathrm{CBP} / \mathrm{p} 300$ in the cellular environment. However, binding affinities for the interaction partners might be different and some of the described $\mathrm{CBP} / \mathrm{p} 300$ protein complexes might be physiologically more significant than others. A comparison of binding affinities is rarely included in the description of new binding partners. This omission has apparently led to a number of reported interactions between ligands and $\mathrm{CBP} / \mathrm{p} 300$ that may be in error or due to non-specific interactions.

$\mathrm{CBP} / \mathrm{p} 300$ contains three cysteine/histidine-rich regions $(\mathrm{C} /$ $\mathrm{H} 1-\mathrm{C} / \mathrm{H} 3$ ), which fold into zinc-binding modules. $\mathrm{C} / \mathrm{H} 1$ corresponds to the TAZ (transcriptional adaptor zinc-binding) 1 domain; TAZ2 is part of the $\mathrm{C} / \mathrm{H} 3$ region [5,6]. Although these domains comprise only approx. 100 residues each, which is small relative to the 2441 residues of the entire human CBP protein, they mediate more than half of the described protein interactions [1]. In contrast with the behaviour of other CBP domains such as KIX (CREB- binding domain of $\mathrm{CBP} / \mathrm{p} 300$ ), which can bind synergistically to two transcriptional activation domains in a ternary complex [7], it appears that only 1:1 complexes are formed by TAZ domains [8]. Several binding partners of $\mathrm{CBP} / \mathrm{p} 300$ have been described as interacting with TAZ1, and structural data are available for complexes formed between TAZ1 and the HIF-1 $\alpha$ (hypoxia-inducible factor $1 \alpha)$ or the CITED2 (CBP/p300-interacting transactivator with glutamic- and aspartic-rich tail) transactivation domain [9-12]. The three zinc ions bound by TAZ stabilize the fold of the domain consisting of four $\alpha$-helices packed against each other. Zinc-depleted TAZ1 is incapable of binding to HIF-1 $\alpha$ [13].

The tumour suppressor p53 is a cellular transcription factor that is up-regulated upon various stress signals including DNA damage. It initiates DNA repair, cell-cycle arrest and/or apoptosis [14-16]. p53 contributes to the stability of the genome, and loss of its function is reported in more than $50 \%$ of human cancers. p53 has a short half life and its cellular level is regulated by MDM2-dependent ubiquitination [17,18] and subsequent degradation by the $25 \mathrm{~S}$ proteasome $[19,20]$. Interaction of the $\mathrm{p} 53$ transactivation domain with the N-terminus of the E3 ubiquitin ligase MDM2 (mouse double minute protein) inhibits p53-dependent transcription [21,22]. The crystal structure of this complex has been described in [23]. p53 binding to MDM2 is weakened by phosphorylation of $\mathrm{Thr}^{18}$ and is slightly enhanced by phosphorylation of $\operatorname{Ser}^{15}$ and $\operatorname{Ser}^{20}$ [24]. Both MDM2 and p53 are described interaction partners of $\mathrm{CBP} / \mathrm{p} 300$. A key role in regulating $\mathrm{p} 53$

Abbreviations used: CBP, cAMP-response-element-binding-protein-binding protein; C/H, cysteine/histidine-rich region; CITED2, CBP/p300-interacting transactivator with glutamic- and aspartic-rich tail; CREB, cAMP-response-element-binding protein; DTT, dithiothreitol; E6-AP, E6-associated protein; GST, glutathione S-transferase; HDM2, human homologue of mouse double minute protein; HIF-1 hypoxia-inducible factor 1; HPV, human papillomavirus; IPTG, isopropyl $\beta$-D-thiogalactoside; MDM2, mouse double minute protein; STAT2, signal transducer and activator of transcription 2; TAZ, transcriptional adaptor zinc-binding domain.

1 To whom correspondence should be addressed (e-mail wright@scripps.edu). 


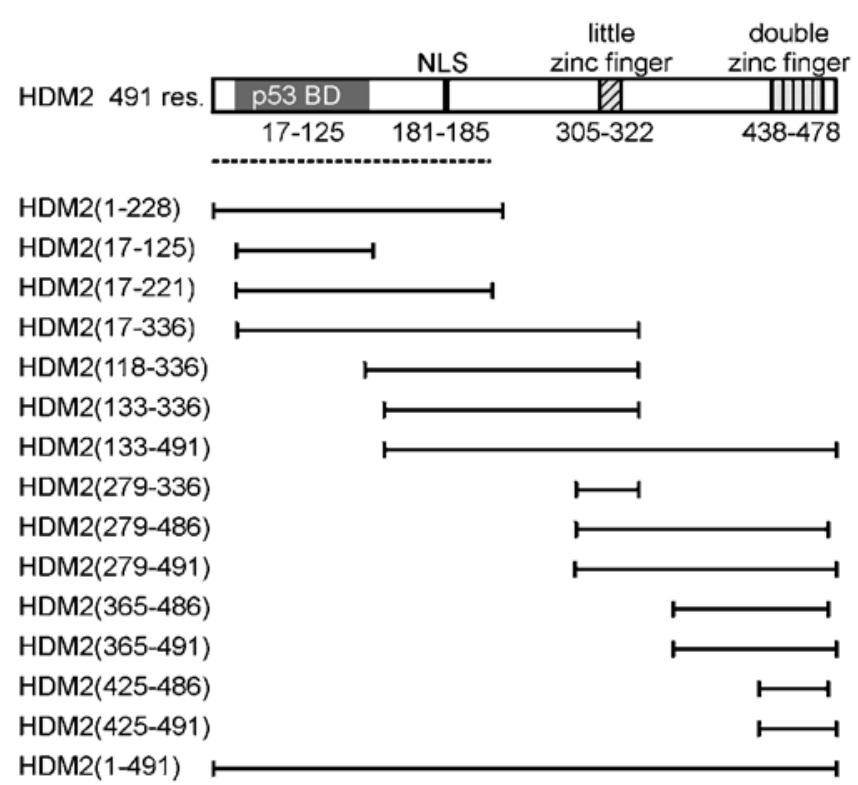

Figure 1 Schematic presentation of the HDM2 domain organization

The p53-binding region is located at the N-terminus (residues 17-125). The nuclear localization signal, the little zinc-finger motif, and a predicted double zinc finger with some similarity to a RING domain are displayed. The proposed p300-interacting region (according to [31]) is indicated with a broken line. The HDM2 fragments used in the present study are shown.

activity was assigned to a C-terminal domain of CBP/p300 [25], and a number of binding sites in $\mathrm{CBP} / \mathrm{p} 300$ for $\mathrm{p} 53$ have been defined, e.g. the TAZ1, KIX (CREB-binding domain of CBP/ p300), C/H2, ZZ (zinc-binding domain in CBP/p300 and dystrophin), TAZ2 and IBiD (interferon-regulatory factor 3-binding domain) domain [26-31].

Grossman and co-workers also proposed a role for $\mathrm{CBP} / \mathrm{p} 300$ in p53 degradation $[26,31,32]$. They suggested that in unstressed cells, the unmodified p53 transactivation domain preferentially binds to MDM2, bound to the TAZ1 domain of CBP/p300 to form a ternary complex. Thus the TAZ1 domain of p300 has been proposed to serve as a scaffold that presents p53 for ubiquitination by MDM2. The proposed p300-binding domain is located within the first 221 residues of MDM2 and a p300 fragment comprising residues 340-406 was shown to bind full-length MDM2 [31]. This proposed MDM2-binding site on p300 contains only part of the well conserved TAZ1 or $\mathrm{C} / \mathrm{H} 1$ domain.

In order to investigate the interaction between a co-activator and an oncoprotein on a structural basis, we expressed and purified various HDM2 (human homologue of MDM2) fragments based on the previously described p300-MDM2 complexes [31], and examined their binding properties for TAZ1 and p53 by CD spectroscopy. The interaction between MDM2 and p53 could be confirmed, but no specific binding was detectable between MDM2 and the CBP TAZ1 domain. Extending our interaction studies to other MDM2 domains (Figure 1) revealed that various regions of MDM2 form non-specific aggregates in solution. These results were confirmed using a GST (glutathione S-transferase)-p300 TAZ1 fusion protein and in vitro translated HDM2 fragments. Unfolded TAZ1 aggregates as well under certain conditions. Mixing the two proteins together leads to the formation of nonnative high-molecular-mass protein complexes with low solubility. Our data clearly demonstrate that TAZ1 in its fully folded native state cannot serve as a specific binding domain for MDM2.

\section{EXPERIMENTAL}

\section{Plasmids}

For expression of recombinant proteins in Escherichia coli, a cDNA encoding HDM2(17-125) was cloned into a bicistronic expression vector [33], together with a fragment encoding the transactivation domain of human p53 (residues 1-61). Murine cDNA encoding CBP(340-439) (the TAZ1 domain) was inserted into pET21a. The corresponding fragment of human p300 (encoding residues 323-424) was cloned into pET42a, directing the synthesis of a GST-TAZ1 fusion protein. GST-p53 (residues 1-61) was constructed by ligation of the corresponding cDNA into a pET42a-derivative. In order to generate templates for in vitro translation, cDNA fragments encoding various domains of HDM2 (see Figure 1) were amplified from a human liver cDNA library by using a PCR-based mutagenesis technique and inserted into pET21a via NdeI/BamHI sites. HIF- $\alpha(705-826)$, CITED2(220 269), STAT2(748-851) (signal transducer and activator of transcription 2), as well as full-length HDM2 cDNA (a gift from Dr Peiqing Sun, Department of Molecular Biology, The Scripps Research Institute) were subcloned into pET21a.

\section{Protein preparation}

CBP TAZ1 (residues 340-439 of mouse CBP) was prepared according to the method used for $\operatorname{mCBP}(345-439)$ [9], and HIF$1 \alpha(790-826)$ was prepared as described in [9]. Purified refolded TAZ1 and HIF- $1 \alpha$ were dialysed into buffer A ( $10 \mathrm{mM}$ Tris/ $\mathrm{HCl}$, pH 7 and $150 \mathrm{mM} \mathrm{NaCl}$ ). HDM2(17-125) was co-expressed with the p53 transactivation domain from a bicistronic vector to enhance protein stability. A growing culture of BL21(DE3) [DNAY] cells harbouring the expression plasmid was induced with $1 \mathrm{mM}$ IPTG (isopropyl $\beta$-D-thiogalactoside) at $37^{\circ} \mathrm{C}$, and protein expression was continued for 3-4 h. Proteins were purified by ionexchange chromatography on Q or SP Sepharose respectively, and subsequent reversed-phase HPLC in a $0.1 \%$ trifluoroacetic acid water/acetonitrile gradient.

HDM2(17-221) was expressed by itself since co-expression with either p53 or TAZ1 did not enhance protein solubility or yield. The protein was purified from the insoluble fraction of E. coli lysates. Briefly, bacterial pellets were suspended in $40 \mathrm{ml}$ of $10 \mathrm{mM}$ Tris/ $\mathrm{HCl}, \mathrm{pH} 6$, per litre of original culture. Bacteria were lysed by lysozyme treatment and subsequent sonication. A DNase I digest was performed and the suspension was adjusted to $0.5 \mathrm{M} \mathrm{NaCl}$ and $2 \%(\mathrm{v} / \mathrm{v})$ Triton X-100. After $30 \mathrm{~min}$ incubation at room temperature $\left(20^{\circ} \mathrm{C}\right)$, the insoluble fraction was isolated by centrifugation at $20000 \mathrm{~g}$ for $30 \mathrm{~min}$. The pellet was washed twice in $20 \mathrm{mM}$ Tris $/ \mathrm{HCl}, \mathrm{pH} 8$, and the inclusion bodies were solubilized in GTD buffer [6 M guanidinium chloride, $20 \mathrm{mM}$ Tris/ $\mathrm{HCl}$,

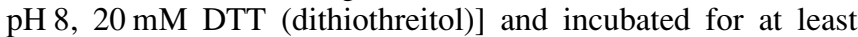
2-4 h. The $\mathrm{pH}$ was lowered by the addition of trifluoroacetic acid and the solution was loaded onto a reversed-phase HPLC column. Elution of the protein was performed in a $0.1 \%$ trifluoroacetic acid water/acetonitrile gradient. HDM2(17-221) containing fractions were freeze-dried and stored at $-20^{\circ} \mathrm{C}$ until use. For CD studies purified HDM2(17-125) or HDM2(17-221) was dissolved in GTD buffer and incubated at room temperature for $2 \mathrm{~h}$. Proteins were refolded on a NAP-5 column (Amersham Biosciences) in buffer A and used for experiments within 1 day. Integrity of the purified proteins was confirmed by MALDI (matrix-assisted laser-desorption ionization) MS.

\section{CD spectroscopy}

Protein concentration was determined by measuring the UV absorbance [34]. Spectra were recorded at $25^{\circ} \mathrm{C}$ at a total protein 
concentration of $10 \mu \mathrm{M}$ in $3 \mathrm{mM}$ Tris/ $\mathrm{HCl}, \mathrm{pH} 7$, and $150 \mathrm{mM}$ $\mathrm{NaCl}$ using a $0.2 \mathrm{~cm}$ cell in an AVIV model $202 \mathrm{CD}$ spectrometer. Proteins used for CD interaction studies were CBP TAZ1, HDM2(17-125) and HDM2(17-221), HIF-1 $\alpha(790-826)$ and p53(1-61). Unfolded CBP TAZ1 or GST-TAZ1 (p300) was obtained by the addition of $2 \mathrm{mM}$ EDTA; for reconstitution attempts, $2.2 \mathrm{mM}$ $\mathrm{ZnSO}_{4}$ was added. The molar ellipticity for the protein solutions was calculated using the total concentration of amino acid residues present.

\section{Preparation of GST fusion proteins and in vitro interaction assay}

Expression plasmids encoding GST-TAZ1 or GST-p53 were transformed into $E$. coli strain BL21(DE3) or BL21(DE3) [DNAY] respectively. Cultures were grown in LB (Luria-Bertani) broth at $37{ }^{\circ} \mathrm{C}$ to a $D_{600}$ of 0.5 . For the expression of GST-TAZ1, $\mathrm{ZnSO}_{4}$ was added to a final concentration of $150 \mu \mathrm{M}$, and the culture was shifted to $25^{\circ} \mathrm{C}$. Recombinant protein synthesis was induced by the addition of IPTG. After 3-4 h of incubation, bacteria were harvested by centrifugation at $6000 \mathrm{~g}$ for $15 \mathrm{~min}$. The pellets were suspended in $200 \mathrm{ml}$ of $20 \mathrm{mM}$ Tris/ $\mathrm{HCl}, \mathrm{pH} 7$, per litre of original culture, and GST-TAZ1-expressing cultures were supplemented with $10 \mu \mathrm{M} \mathrm{ZnSO}_{4}$. The suspensions were stored at $-20{ }^{\circ} \mathrm{C}$ until use. For purification of GST fusion proteins, $40 \mathrm{ml}$ aliquots were thawed at room temperature, supplemented with protease inhibitors (Complete ${ }^{\mathrm{TM}}$, EDTA-free; Roche Diagnostics) and $1 \mathrm{mM}$ DTT. Bacteria were lysed using a French press and GST fusion proteins were pre-purified by ion-exchange chromatography. Fractions containing the fusion protein were supplemented with protease inhibitors and stored at $4{ }^{\circ} \mathrm{C}$ for up to 3 weeks. For the in vitro interaction assay, approx. $5 \mu \mathrm{g}$ of GST fusion protein was bound to $2.5 \mu \mathrm{l}$ of glutathione-Sepharose and washed three times in wash buffer $(20 \mathrm{mM}$ Tris/ $\mathrm{HCl}, \mathrm{pH} 7$, $150 \mathrm{mM} \mathrm{NaCl}, 1 \mathrm{mM}$ DTT, $0.1 \%$ (v/v) Nonidet P40, Complete ${ }^{\mathrm{TM}}$ protease inhibitors and additional $10 \mu \mathrm{M} \mathrm{ZnSO}_{4}$ for GST-TAZ1).

${ }^{35} \mathrm{~S}$-labelled proteins were in vitro translated using wheat germ lysates (Promega) according to the manufacturer's protocol. To minimize RNA and protein degradation RNasin (Promega) and protease inhibitors were added to the reaction mixture. The in vitro translation product $(1.5 \mu \mathrm{l})$ was mixed with protein sample buffer and loaded to the gel directly (10\% input), $15 \mu \mathrm{l}$ of the in vitro translation product was diluted in $150 \mu \mathrm{l}$ of wash buffer and added to the Sepharose-bound fusion proteins. After $20 \mathrm{~min}$ of mixing at room temperature, the resins were washed three times in $150 \mu \mathrm{l}$ of wash buffer. To compare binding affinities of TAZ1 in its native or its unfolded state, parallel reactions were performed using wash buffer containing $2 \mathrm{mM}$ EDTA instead of $\mathrm{ZnSO}_{4}$. The washed resin was either boiled directly in protein sample buffer (unfolded TAZ1) or soluble protein complexes were eluted in $100 \mu \mathrm{l}$ of wash buffer containing additional $25 \mathrm{mM}$ reduced glutathione (native TAZ1 or p53). Eluates were concentrated by precipitation with $10 \%$ trichloroacetic acid. Protein pellets were dissolved in sample buffer and loaded on to a 4-20\% acrylamide gel for SDS/PAGE. Binding of ${ }^{35} \mathrm{~S}$-labelled proteins was detected by autoradiography.

\section{RESULTS}

\section{Protein preparation and CD spectroscopy}

To investigate the interaction between HDM2 and CBP/p300 on a structural basis, N-terminal HDM2 fragments and the CBP TAZ1 domain were expressed in E. coli and purified to homogeneity. Proteins were refolded and analysed for secondary-structure content and protein-binding properties by CD spectroscopy. HDM2(17-221) displays some $\alpha$-helical structure as exhibited by the typical absorption bands at 222 and $208 \mathrm{~nm}$ (Figure 2A). Free TAZ1 also folds into a helical structure. In the present study, the CD spectrum obtained for a mixture of HDM2 and TAZ1 did not differ from a curve calculated from data obtained for the two isolated domains (broken line). This suggests either that the formation of the protein complex is not accompanied by additional secondary structure formation or that there is no interaction between the two proteins.

In order to confirm the native fold of the HDM2 and the TAZ1 protein preparations, control experiments were performed with HDM2 and p53, as well as with TAZ1 and HIF- $1 \alpha$. The transactivation domain of p53 is unfolded in solution (Figure $2 \mathrm{~B}$ ). The CD spectrum of a 1:1 mixture of this protein with HDM2(17221) differed from the calculated average of the spectrum of the two free proteins (broken line). A similar result was obtained for a mixture of HDM2(17-125) and p53 (Figure 2D). The biological activity and the structural integrity of the refolded CBP TAZ1 domain was confirmed by its ability to interact with HIF- $1 \alpha$. Free HIF- $1 \alpha(790-826)$ is unfolded in solution as well (Figure 2C). However, a 1:1 mixture with TAZ1 displays a higher $\alpha$-helical content than the calculated average of the two free proteins (broken lines), suggesting additional secondary-structure elements in the complex. Indeed, binding of HIF- $1 \alpha(790-826)$ to TAZ1 is accompanied by the formation of two helical regions (termed $\alpha_{\mathrm{B}}$ and $\alpha_{\mathrm{C}}$ ) in the 37-residue HIF- $1 \alpha$ peptide spanning nine and ten residues respectively $[9,11]$. Therefore the TAZ1HIF complex (137 residues) gains a calculated 14\% helicity compared with the isolated domains, which is reflected in our CD results. The N-terminal domain of p53 in its free state is unfolded as well (Figures 2B and 2D; and [35,36]. Within the 61 residues of the p53 peptide only nine may fold into a helix [23]. Thus a complex formed between p53(1-61) and HDM2(17-125) or HDM2(17-221) gains only $5 \%$ or $3 \%$ helicity respectively. This explains the fact that the difference between the calculated and the measured spectrum is bigger for TAZ1-HIF complexes than for HDM2-p53 complexes.

The molar ellipticity of HDM2(17-221) is approximately half of the ellipticity of HDM2(17-125), and the protein is about double in size (205 residues compared with 109). Since this property is an average over all peptide bonds, this suggests that in free HDM2(17-221), approximately the same number of residues are folded as in free HDM2(17-125). Thus the region beyond residue 125 may be unfolded, and one would expect that it forms some secondary-structure elements upon interaction with TAZ1. However, the primary sequence of the HDM2 central region displays low complexity and is rich in proline and serine residues, which is common for unstructured segments. In contrast, the amino acid sequences of the HIF- $1 \alpha$ and CITED2 TAZ1-interaction domains are more complex and structural prediction reveals a significant helical content for the binding region. Despite the transactivation domains of HIF- $1 \alpha$ and CITED2 being unfolded in their free state, they fold into helical structures upon binding to TAZ1 (Figure 2C; and $[9,11,12]$ ).

Previously, it has been shown that phosphorylation of HDM2 on $\operatorname{Ser}^{166}$ and Ser $^{186}$ enhances its interaction with p300 [37]. We prepared HDM2(Ser166Asp), HDM2(Ser186Asp) and HDM2(Ser166Asp,186Asp) in order to mimic these modifications in our recombinant protein. Neither of the mutant HDM2 proteins displayed an interaction with TAZ1 (results not shown).

To examine the influence of zinc binding on the secondarystructure content of TAZ1, a folded CBP TAZ1 protein preparation (Figure 2E) was treated with EDTA, resulting in a spectrum of an unfolded protein. Addition of an excess of $\mathrm{Zn}^{2+}$ did not restore the native fold, suggesting that there is no equilibrium between the zinc-bound and the zinc-depleted form of TAZ1. A natively 
A

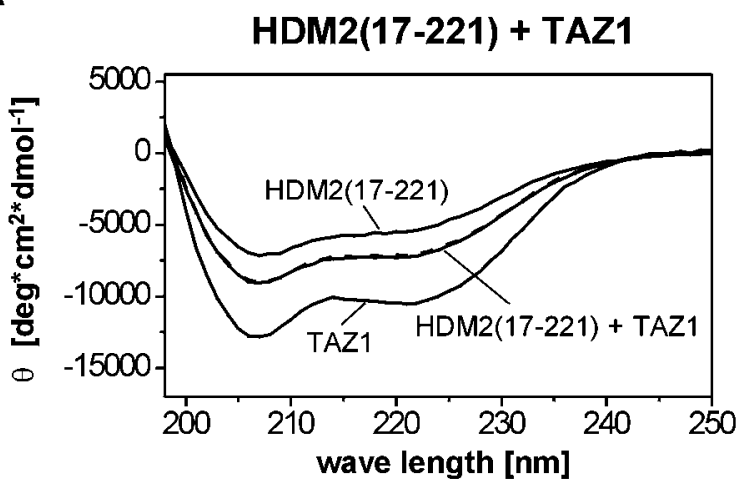

C

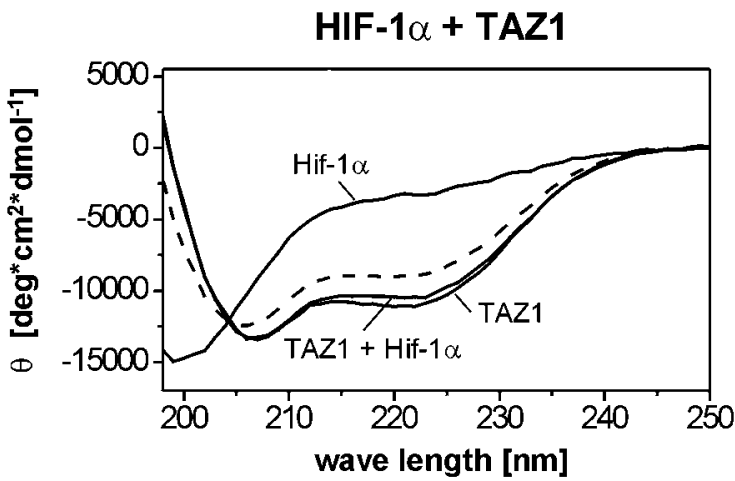

E

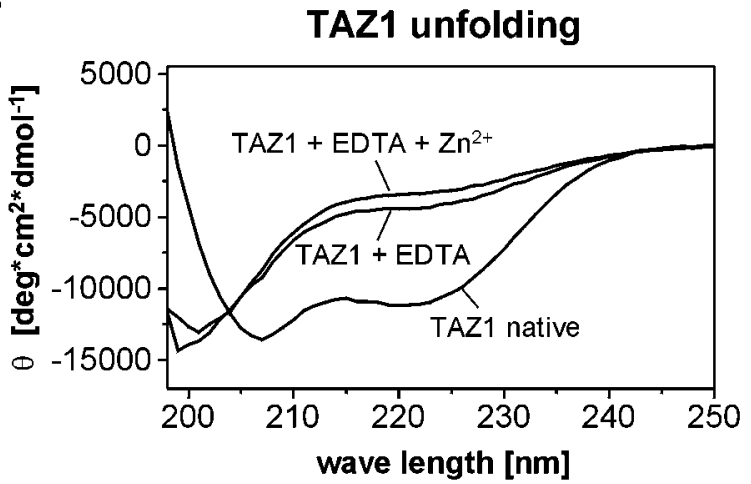

B

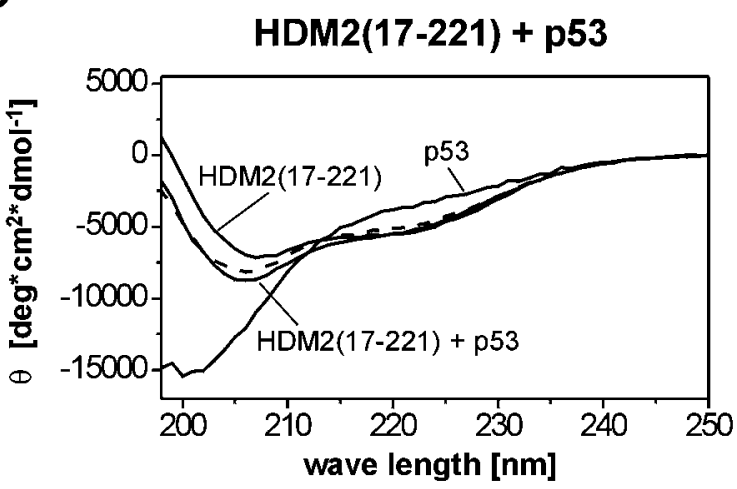

D

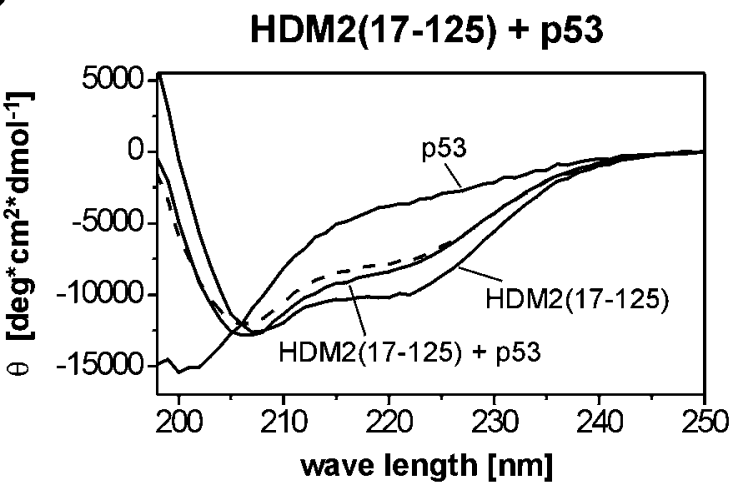

F

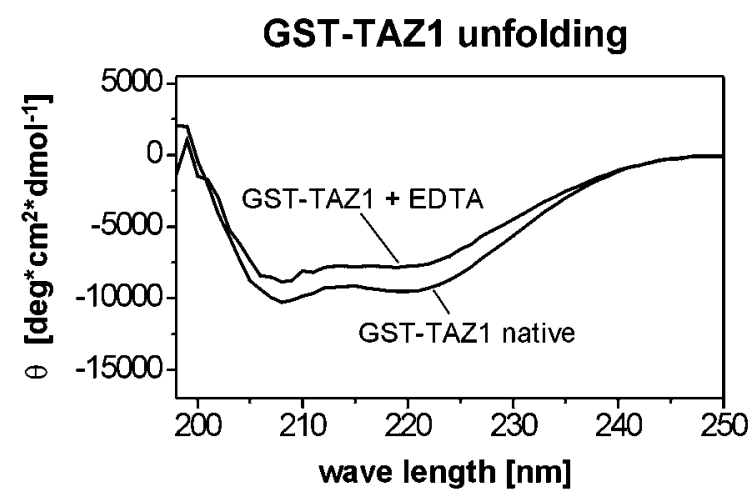

Figure 2 Far UV CD spectroscopy to monitor protein-protein interaction and unfolding

Wavelength scans for the indicated proteins are displayed in solid lines, the calculated average of the molar ellipticity of two free proteins is shown in broken lines. (A) CBP-TAZ1 and HDM2(17-221) were refolded after denaturing purification. Both proteins exhibit $\alpha$-helical structure, although the helix content is much higher in TAZ1 than in HDM2(17-221). The spectrum of the mixture of the two proteins is identical with the calculated average curve. (B) Recombinant purified p53(1-61) is unfolded in its free state, but interacts with refolded HDM2(17-221). The spectrum of a mixture with HDM2(17-221) differs slightly from the calculated average of the two isolated domains. (C) The native fold of the free TAZ1 protein and the ability to interact with HIF-1 $\alpha$ (790-826) was confirmed. (D) Interaction of HDM2(17-125) with p53(1-61) confirming the described p53-binding site in the N-terminus of HDM2. (E) Unfolding of CBP TAZ1 upon treatment with a chelating agent. The native helical structure of the zinc-binding domain is destroyed by the addition of EDTA and could not be restored by addition of an excess of zinc. (F) The p300 TAZ1 domain fused to GST (GST-TAZ1) loses helical structure after treatment with EDTA, suggesting unfolding of the zinc-binding domain.

purified GST fusion protein, which contains the p300 TAZ1 domain (GST-TAZ1), exhibited similar behaviour. The $\alpha$-helical content of the protein (Figure $2 \mathrm{~F}$ ) decreased significantly after addition of EDTA. Assuming that the four helices of TAZ1 are formed in the native GST fusion protein (380 residues), it loses $16 \%$ helicity upon EDTA treatment. This is reflected by our results and indicates unfolding of the zinc-binding domain, whereas the secondary structure elements of GST are stable under these conditions.

\section{GST in vitro interaction assay}

CD spectroscopy might not be sensitive enough to detect small structural changes in relatively large protein complexes, and the proteins used for the studies were purified from E. coli lysates. The interaction between TAZ1 and HDM2 was first proposed based on pull down assays using bacterially expressed GST-p300 TAZ1 fusion proteins together with in vitro translated HDM2 peptides [31]. In order to enhance the sensitivity of our 


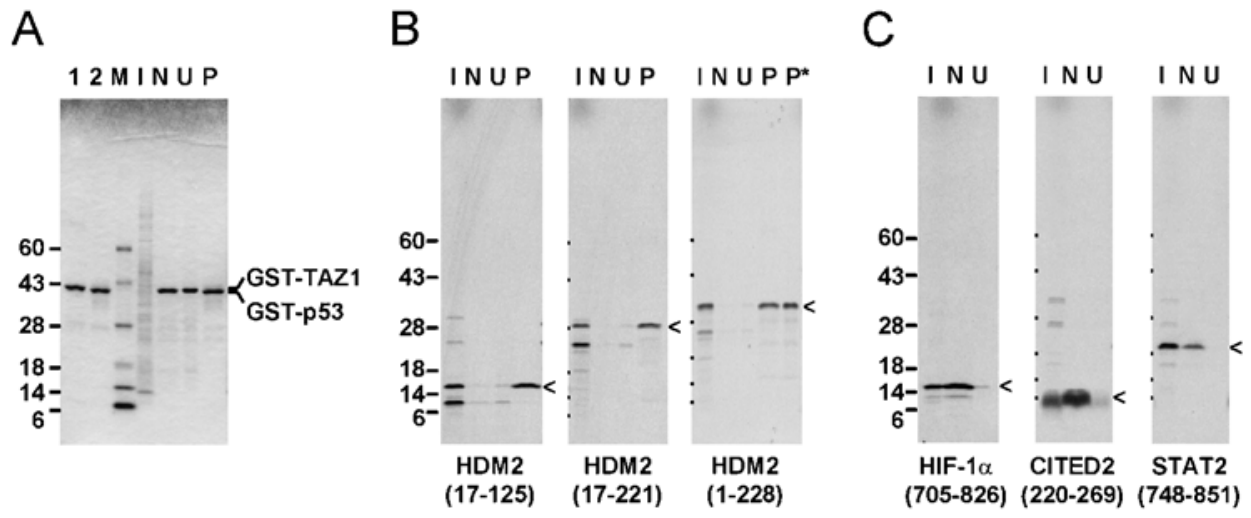

\section{Figure 3 GST in vitro interaction assay}

For all gels, the same size standards were used, the position of the bands are indicated in the margin or labelled by dots on each panel. (A) SDS/PAGE of purified GST-TAZ1 and GST-p53 (lanes 1 and 2 respectively). M, molecular mass marker; I, $10 \%$ of the input of a typical in vitro reaction. Sepharose-bound fusion proteins were incubated with in vitro translated ${ }^{35}$ S-labelled peptides. Soluble complexes formed with native GST-TAZ1 (N) or GST-p53 (P) were eluted with an excess of glutathione, whereas unfolded aggregates were solubilized in sample buffer (U). Proteins were analysed by SDS/PAGE. (B and C) Autoradiography of bound ${ }^{35}$ S-labelled proteins, lanes are labelled as in (A). Exposure times varied between 16 and $48 \mathrm{~h}$, but were always the same within one in vitro experiment. (B) Binding of ${ }^{35} \mathrm{~S}$-labelled in vitro translated N-terminal fragments of HDM2 to GST-p53 and GST-TAZ1 under native and denaturing conditions. The biological activity of the in vitro translated HDM2 protein fragments was confirmed by interaction with GST-p53 [e.g. HDM2(17-125), HDM2(17-221) and HDM2(1-228)]. As expected, addition of EDTA to GST-p53(1-61) did not affect the interaction with HDM2(1-228) (lanes P and P*). No specific HDM2-TAZ1 interaction could be detected under native conditions. (C) The known TAZ1-binding partners HIF-1 $\alpha$, CITED2 and STAT2 were used as positive controls. Their interaction with TAZ1 is strong and depends on a native fold (compare lanes $\mathrm{N}$ and U).

interaction studies, we decided to use the same technique as an additional tool. Furthermore, in vitro translated HDM2 peptides may be post-translationally modified, which may be required for interaction. The TAZ1 domains of CBP and p300 share high homology; within the structured regions, $96 \%$ of the amino acid residues are identical [8]. Although some interacting proteins, e.g. HIF- $1 \alpha$ or CITED2, are known to bind to the TAZ1 domain of both CBP and p300 [9-12], one cannot exclude the possibility that there might be some differences in binding specificity. To answer the question of whether the described MDM2 interaction is specific for one of the paralogues, we used the TAZ1 domain of human p300 fused to GST for interaction studies together with in vitro translated HDM2. Control experiments for HDM2 were performed with a GST fusion protein containing the transactivation domain of p53. Both GST-TAZ1 and GST-p53 were purified from the soluble fraction of $E$. coli lysates under native conditions using ion-exchange and affinity chromatography (Figure 3A, lanes 1 and 2 respectively). Fusion proteins immobilized on glutathione-Sepharose were incubated with in vitro translated ${ }^{35} \mathrm{~S}$-labelled HDM2 proteins. Native wash conditions were applied for GST-TAZ1 and GST-p53 (labelled N or P respectively in Figure 3). Zinc-chelating wash conditions were applied for parallel reactions with GST-TAZ1 (labelled U in Figure 3) to distinguish between folded protein complexes and non-specific aggregates formed by unfolded (zinc-free) TAZ1. To confirm the efficiency of the zinc-chelation, a control experiment was performed. Bacterially expressed immobilized GST-HIF1 $\alpha$ was incubated with in vitro translated TAZ1. Under native conditions, TAZ1 bound to the GST-HIF protein, but the interaction was abolished when zinc-chelating wash conditions were applied (results not shown). The proteins were either eluted from the resin in a native buffer (lanes $\mathrm{N}$ and $\mathrm{P}$ ) or the resin was boiled in denaturing sample buffer (lane U). These elution techniques were applied to achieve equal protein yields under native and non-native conditions as monitored by SDS/PAGE (compare lanes $\mathrm{N}$ and $\mathrm{U}$ in Figure 3A). Affinity of the various ${ }^{35}$ S-labelled domains for p53 and/or native and unfolded TAZ1 was estimated by autoradiography and comparison with $10 \%$ of the input (lanes I in Figure 3) of the in vitro reactions.
HDM2(17-125), HDM2(17-221) and HDM2(1-228) displayed strong interaction with GST-p53 (Figure 3B) in agreement with published data $[23,38]$. As expected, addition of EDTA did not affect complex formation between p53 and HDM2 (Figure 3B, compare lanes $\mathrm{P}$ and $\mathrm{P}^{*}$ in the third panel). Neither HDM2(17221) nor HDM2(1-228) bound to GST-TAZ1, which is in contrast with results from previous mapping experiments where HDM2(1$325)$ and $\operatorname{HDM} 2 \Delta(222-350)$ bound to the N-terminus of p300 [31]. Control experiments with in vitro translated HIF-1 $\alpha(705-$ 826), CITED2(220-269) and STAT2(748-851) confirmed the biological activity and the structural integrity of the GST-fused TAZ1 domain. All three proteins bound strongly to TAZ1 under native conditions in agreement with published data $[13,39,40]$, but no interaction was detected with zinc-depleted, unfolded TAZ1 (Figure 3C).

In order to extend our interaction study to the entire HDM2 protein, the full-length HDM2 protein and fragments beyond the p53-binding region were examined for specific TAZ1 binding. HDM2(1-491) and HDM2(133-491) showed weak binding to native TAZ1, but exhibited at least the same or stronger affinity for the denatured zinc domain (Figure 4A). Fragments containing the central acidic region and/or the little zinc finger did not recognize TAZ1 under native conditions either (Figure 4B). However, some HDM2 constructs, e.g. HDM2(17-336), HDM2(118-336) and HDM2(133-336) bound strongly to unfolded TAZ1. The same behaviour was observed for HDM2 peptides spanning the region from the little zinc finger to the C-terminus (Figure 4C). None of these HDM2 constructs bound specifically to the folded TAZ1 domain, but some displayed weak interaction under denaturing conditions. Deletion of the five C-terminal amino acid residues (Leu-Thr-Tyr-Phe-Pro), which contain hydrophobic bulky side chains, improved the solubility of the peptides and prevented the non-specific aggregation. Similarly, large unstructured regions might contribute to the aggregation tendency of bigger HDM2 fragments, even at the low concentration used in our interaction assay. p53 interaction stabilizes only residues 17-125 of HDM2. In the present study, co-expression of these two peptides in E. coli yielded a soluble complex. Bacterially expressed HDM2(17221) however was insoluble, irrespective of whether it was 
A

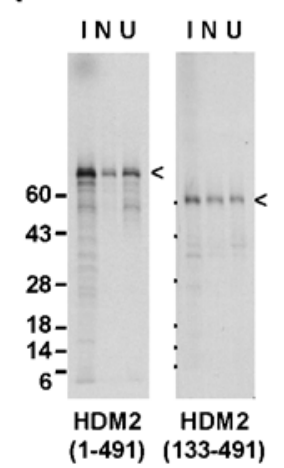

B

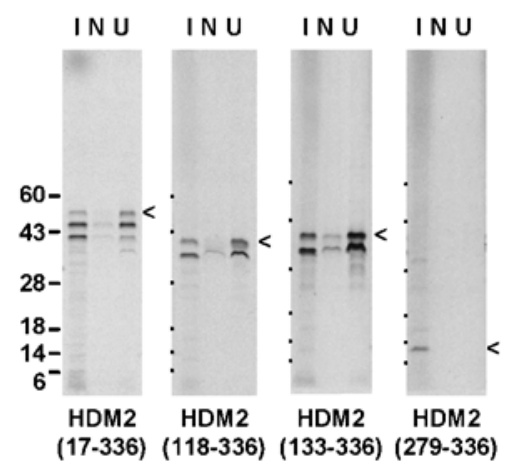

C

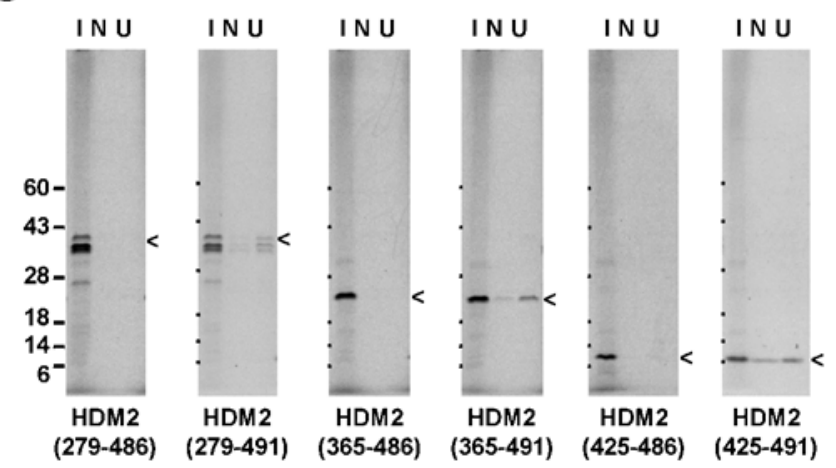

binding that was detectable in some cases by the affinity chromatography technique used.

GST-TAZ1 in its native state bound to the described interaction partners HIF- $1 \alpha$, CITED2 and STAT2 (Figure 3C). In contrast, the partly unfolded fusion protein lost this ability, suggesting that these protein-protein interactions depend on a natively folded TAZ1 domain and are highly specific. This is in agreement with reports of the zinc-dependence of HIF- $1 \alpha$ and CITED2 TAZ1 complex formation $[10,13]$. The weak binding of GST-TAZ1 to some HDM2 fragments, however, was enhanced after EDTA treatment, demonstrating that the observed CBP/p300 TAZ1 MDM2 interaction [31] is independent of a native fold of the zinc domain and is therefore non-specific. Interestingly, screening for GST$\mathrm{C} / \mathrm{H} 1$ binding partners using an expression library derived from HeLa cells resulted in the isolation of clones for CITED2, HIF- $1 \alpha$ and STAT2 [39], but not for HDM2, although the corresponding cDNA clone was previously isolated from a cDNA library derived from the same cell line [38].

Initially, the HDM2-TAZ1 interaction was described based on some sequence similarity between two regions of the "high risk' HPV (human papillomavirus) E6 protein and a C-terminal region of $\mathrm{CBP} / \mathrm{p} 300$ and the first zinc cluster of the TAZ1 domain respectively [31]. HPV E6 in complex with the cellular E6-AP (E6-associated protein) also targets p53 for ubiquitin-dependent proteolysis [41,42]. However, this occurs exclusively in HPVinfected cells $[43,44]$. Several other differences between the two p53-degradation mechanisms have been defined [45,46]. E6 recognizes the core domain of p53, and not the N-terminus as HDM 2 does $[47,48]$. HDM2 contains the p53 and the ubiquitinrecognition element on the same polypeptide, whereas the corresponding domains are linked by protein-protein interactions in the case of E6/E6-AP. Thus it is very unlikely that the sequence similarity between E6 and TAZ1 has functional significance in the degradation pathway of p53.

Unfortunately, many interaction studies including zinc-binding domains are performed in the presence of EDTA, because it is commonly used as an inhibitor of metalloproteases. Complex formation between MDM2 and the CBP/p300 TAZ1 domain has been reported by several other research groups [31,49-53], but their experimental conditions all include EDTA, which indicates that they are working with denatured $\mathrm{CBP} / \mathrm{p} 300$ domains. When EDTA is excluded from the medium, as in the present study, no interaction is observed between the correctly folded CBP/p300 TAZ1 (C/H1) domain and HDM2. Either the interaction between $\mathrm{CBP} / \mathrm{p} 300$ and HDM2 involves a different domain of the coactivator or it is mediated by other proteins. Thus the role of the central acidic domain of MDM2 in p53 ubiquitination and the mechanism by which overexpression of the TAZ1 domain enhances p53 stability still awaits clarification.

We acknowledge Jonathan Wojciak for helpful discussion. T.M. thanks the Austrian Science Foundation (FWF) for support by Erwin Schrödinger Fellowships J2137 and J2292. This work was supported by grant CA96865 from the National Institutes of Health and by the Skaggs Institute for Chemical Biology.

\section{REFERENCES}

1 Goodman, R. H. and Smolik, S. (2000) CBP/p300 in cell growth, transformation, and development. Genes Dev. 14, 1553-1577

2 Janknecht, R. and Hunter, T. (1996) Transcription: a growing coactivator network. Nature (London) 383, 22-23

3 Giles, R. H., Peters, D. J. and Breuning, M. H. (1998) Conjunction dysfunction: $\mathrm{CBP} / \mathrm{p} 300$ in human disease. Trends Genet. 14, 178-183

4 Shikama, N., Lyon, J. and LaThangue, N. B. (1997) The p300/CBP family: integrating signals with transcription factors and chromatin. Trends Cell Biol. 7, 230-236 
5 De Guzman, R. N., Liu, H. Y., Martinez-Yamout, M., Dyson, H. J. and Wright, P. E. (2000) Solution structure of the TAZ2 (CH3) domain of the transcriptional adaptor protein CBP. J. Mol. Biol. 303, 243-253

6 Ponting, C. P., Blake, D. J., Davies, K. E., Kendrick-Jones, J. and Winder, S. J. (1996) ZZ and TAZ: new putative zinc fingers in dystrophin and other proteins. Trends Biochem. Sci. 21, 11-13

7 Goto, N. K., Zor, T., Martinez-Yamout, M., Dyson, H. J. and Wright, P. E. (2002) Cooperativity in transcription factor binding to the coactivator CREB-binding protein (CBP): the mixed lineage leukemia protein (MLL) activation domain binds to an allosteric site on the KIX domain. J. Biol. Chem. 277, 43168-43174

8 De Guzman, R. N., Martinez-Yamout, M. A., Dyson, H. J. and Wright, P. E. (2004) Structure and function of the CBP/p300 TAZ domains. In Zinc Finger Proteins: from Atomic Contact to Cellular Function (luchi, S. and Kuldell, N., eds.), in the press, Landes Bioscience, Georgetown

9 Dames, S. A., Martinez-Yamout, M., De Guzman, R. N., Dyson, H. J. and Wright, P. E. (2002) Structural basis for Hif-1 $\alpha /$ CBP recognition in the cellular hypoxic response. Proc. Natl. Acad. Sci. U.S.A. 99, 5271-5276

10 Freedman, S. J., Sun, Z. Y., Kung, A. L., France, D. S., Wagner, G. and Eck, M. J. (2003) Structural basis for negative regulation of hypoxia-inducible factor- $1 \alpha$ by CITED2. Nat. Struct. Biol. 10, 504-512

11 Freedman, S. J., Sun, Z. Y., Poy, F., Kung, A. L., Livingston, D. M., Wagner, G. and Eck, M. J. (2002) Structural basis for recruitment of CBP/p300 by hypoxia-inducible factor-1 $\alpha$. Proc. Natl. Acad. Sci. U.S.A. 99, 5367-5372

12 De Guzman, R. N., Martinez-Yamout, M. A., Dyson, H. J. and Wright, P. E. (2004) Interaction of the TAZ1 domain of the CREB-binding protein with the activation domain of CITED2: regulation by competition between intrinsically unstructured ligands for non-identical binding sites. J. Biol. Chem. 279, 3042-3049

13 Kung, A. L., Wang, S., KIco, J. M., Kaelin, W. G. and Livingston, D. M. (2000) Suppression of tumor growth through disruption of hypoxia-inducible transcription. Nat. Med. 6, 1335-1340

14 Levine, A. J. (1997) p53, the cellular gatekeeper for growth and division. Cell 88, 323-331

15 Sionov, R. V. and Haupt, Y. (1999) The cellular response to p53: the decision between life and death. Oncogene 18,6145-6157

16 Vousden, K. H. and Lu, X. (2002) Live or let die: the cell's response to p53. Nat. Rev. Cancer 2, 594-604

17 Fang, S., Jensen, J. P., Ludwig, R. L., Vousden, K. H. and Weissman, A. M. (2000) Mdm2 is a RING finger-dependent ubiquitin protein ligase for itself and p53. J. Biol. Chem. 275 8945-8951

18 Honda, R., Tanaka, H. and Yasuda, H. (1997) Oncoprotein MDM2 is a ubiquitin ligase E3 for tumor suppressor p53. FEBS Lett. 420, 25-27

19 Haupt, Y., Maya, R., Kazaz, A. and Oren, M. (1997) Mdm2 promotes the rapid degradation of p53. Nature (London) 387, 296-299

20 Kubbutat, M. H., Jones, S. N. and Vousden, K. H. (1997) Regulation of p53 stability by Mdm2. Nature (London) 387, 299-303

21 Chen, J., Lin, J. and Levine, A. J. (1995) Regulation of transcription functions of the p53 tumor suppressor by the mdm-2 oncogene. Mol. Med. 1, 142-152

22 Momand, J., Zambetti, G. P., Olson, D. C., George, D. and Levine, A. J. (1992) The mdm-2 oncogene product forms a complex with the p53 protein and inhibits p53-mediated transactivation. Cell 69, 1237-1245

23 Kussie, P. H., Gorina, S., Marechal, V., Elenbaas, B., Moreau, J., Levine, A. J. and Pavletich, N. P. (1996) Structure of the MDM2 oncoprotein bound to the p53 tumor suppressor transactivation domain. Science 274, 948-953

24 Schon, 0., Friedler, A., Bycroft, M., Freund, S. M. and Fersht, A. R. (2002) Molecular mechanism of the interaction between MDM2 and p53. J. Mol. Biol. 323, 491-501

25 Gu, W., Shi, X. L. and Roeder, R. G. (1997) Synergistic activation of transcription by CBP and p53. Nature (London) 387, 819-823

26 Grossman, S. R. (2001) p300/CBP/p53 interaction and regulation of the p53 response. Eur. J. Biochem. 268, 2773-2778

27 Song, C. Z., Keller, K., Chen, Y., Murata, K. and Stamatoyannopoulos, G. (2002) Transcription coactivator CBP has direct DNA binding activity and stimulates transcription factor DNA binding through small domains. Biochem. Biophys. Res. Commun. 296, 118-124

28 Avantaggiati, M. L., Ogryzko, V., Gardner, K., Giordano, A., Levine, A. S. and Kelly, K. (1997) Recruitment of p300/CBP in p53-dependent signal pathways. Cell 89, 1175-1184

29 Van Orden, K., Giebler, H. A., Lemasson, I., Gonzales, M. and Nyborg, J. K. (1999) Binding of $p 53$ to the KIX domain of CREB binding protein: a potential link to human T-cell leukemia virus, type I-associated leukemogenesis. J. Biol. Chem. 274, 26321-26328
30 Livengood, J. A., Scoggin, K. E., Van Orden, K., McBryant, S. J., Edayathumangalam, R. S., Laybourn, P. J. and Nyborg, J. K. (2002) p53 transcriptional activity is mediated through the SRC1-interacting domain of CBP/p300. J. Biol. Chem. 277, 9054-9061

31 Grossman, S. R., Perez, M., Kung, A. L., Joseph, M., Mansur, C., Xiao, Z. X., Kumar, S., Howley, P. M. and Livingston, D. M. (1998) p300/MDM2 complexes participate in MDM2-mediated p53 degradation. Mol. Cell 2, 405-415

32 Grossman, S. R., Deato, M. E., Brignone, C., Chan, H. M., Kung, A. L., Tagami, H., Nakatani, Y. and Livingston, D. M. (2003) Polyubiquitination of p53 by a ubiquitin ligase activity of $p 300$. Science $\mathbf{3 0 0}, 342-344$

33 Demarest, S. J., Martinez-Yamout, M., Chung, J., Chen, H., Xu, W., Dyson, H. J., Evans, R. M. and Wright, P. E. (2002) Mutual synergistic folding in recruitment of CBP/p300 by p160 nuclear receptor coactivators. Nature (London) 415, 549-553

34 Gill, S. C. and von Hippel, P. H. (1989) Calculation of protein extinction coefficients from amino acid sequence data. Anal. Biochem. 182, 319-326

35 Bell, S., Klein, C., Muller, L., Hansen, S. and Buchner, J. (2002) p53 contains large unstructured regions in its native state. J. Mol. Biol. 322, 917-927

36 Dawson, R., Mueller, I., Dehner, A., Klein, C., Kessler, H. and Buchner, J. (2003) The $\mathrm{N}$-terminal domain of $\mathrm{p53}$ is natively unfolded. J. Mol. Biol. 332, 1131-1141

37 Zhou, B. P., Liao, Y., Xia, W., Zou, Y., Spohn, B. and Hung, M. C. (2001) HER-2/neu induces p53 ubiquitination via Akt-mediated MDM2 phosphorylation. Nat. Cell Biol. 3 973-982

38 Chen, J., Marechal, V. and Levine, A. J. (1993) Mapping of the p53 and mdm-2 interaction domains. Mol. Cell Biol. 13, 4107-4114

39 Bhattacharya, S., Michels, C. L., Leung, M. K., Arany, Z. P., Kung, A. L. and Livingston D. M. (1999) Functional role of p35srj, a novel p300/CBP binding protein, during transactivation by HIF-1. Genes Dev. 13, 64-75

40 Bhattacharya, S., Eckner, R., Grossman, S., Oldread, E., Arany, Z., D'Andrea, A. and Livingston, D. M. (1996) Cooperation of Stat2 and p300/CBP in signalling induced by interferon- $\alpha$. Nature (London) 383, 344-347

41 Scheffner, M., Werness, B. A., Huibregtse, J. M., Levine, A. J. and Howley, P. M. (1990) The E6 oncoprotein encoded by human papillomavirus types 16 and 18 promotes the degradation of $p 53$. Cell 63, 1129-1136

42 Scheffner, M., Huibregtse, J. M., Vierstra, R. D. and Howley, P. M. (1993) The HPV-16 E6 and E6-AP complex functions as a ubiquitin-protein ligase in the ubiquitination of p53. Cell 75, 495-505

43 Beer-Romero, P., Glass, S. and Rolfe, M. (1997) Antisense targeting of E6AP elevates p53 in HPV-infected cells but not in normal cells. Oncogene 14, 595-602

44 Talis, A. L., Huibregtse, J. M. and Howley, P. M. (1998) The role of E6AP in the regulation of p53 protein levels in human papillomavirus (HPV)-positive and HPV-negative cells. J. Biol. Chem. 273, 6439-6445

45 Nakamura, S., Roth, J. A. and Mukhopadhyay, T. (2002) Multiple lysine mutations in the C-terminus of p53 make it resistant to degradation mediated by MDM2 but not by human papillomavirus E6 and induce growth inhibition in MDM2-overexpressing cells. Oncogene 21, 2605-2610

46 Camus, S., Higgins, M., Lane, D. P. and Lain, S. (2003) Differences in the ubiquitination of $\mathrm{p} 53$ by Mdm2 and the HPV protein E6. FEBS Lett. 536, 220-224

$47 \mathrm{Li}, \mathrm{X}$. and Coffino, P. (1996) High-risk human papillomavirus E6 protein has two distinct binding sites within $\mathrm{p53}$, of which only one determines degradation. J. Virol. 70 , 4509-4516

48 Gu, J., Rubin, R. M. and Yuan, Z. M. (2001) A sequence element of p53 that determines its susceptibility to viral oncoprotein-targeted degradation. Oncogene $\mathbf{2 0}$, 3519-3527

49 Wadgaonkar, R. and Collins, T. (1999) Murine double minute (MDM2) blocks p53-coactivator interaction, a new mechanism for inhibition of p53-dependent gene expression. J. Biol. Chem. 274, 13760-13767

50 Kobet, E., Zeng, X., Zhu, Y., Keller, D. and Lu, H. (2000) MDM2 inhibits p300-mediated p53 acetylation and activation by forming a ternary complex with the two proteins. Proc. Natl. Acad. Sci. U.S.A. 97, 12547-12552

51 Jin, Y., Zeng, S. X., Dai, M. S., Yang, X. J. and Lu, H. (2002) MDM2 inhibits PCAF (p300/CREB-binding protein-associated factor)-mediated p53 acetylation. J. Biol. Chem. 277, 30838-30843

52 Zeng, X., Chen, L., Jost, C. A., Maya, R., Keller, D., Wang, X., Kaelin, Jr, W. G., Oren, M., Chen, J. and Lu, H. (1999) MDM2 suppresses p73 function without promoting p73 degradation. Mol. Cell Biol. 19, 3257-3266

53 Kadakia, M., Brown, T. L., McGorry, M. M. and Berberich, S. J. (2002) MdmX inhibits Smad transactivation. Oncogene 21, 8776-8785 